\title{
A trace formula for non-unitary representations of a uniform lattice
}

\author{
Anton Deitmar $\quad$ Frank Monheim
}

\begin{abstract}
In this work we shall generalize the Selberg trace formula to a nonunitary finite-dimensional complex representation $\chi: \Gamma \rightarrow \mathrm{GL}(V)$ of a uniform lattice $\Gamma$ of a real Lie group $G$.
\end{abstract}

\section{Contents}

1 Introduction $\quad 1$

2 Preliminaries

3 The spectral decomposition of the Casimir operator 8

4 The trace formula $\quad 16$

\section{Introduction}

Given a Lie group $G$, a uniform lattice $\Gamma \subset G$ and a finite dimensional unitary representation $\chi: G \rightarrow \mathrm{GL}(V)$, consider the Hilbert space $L^{2}(\Gamma \backslash G, \chi)$ of measurable functions $f: G \rightarrow V$ that satisfy $f(\gamma g)=\chi(\gamma) f(g)$ for all $\gamma \in \Gamma$ and almost everywhere in $g \in G$, with the extra condition, that

$$
\int_{\Gamma \backslash G}\langle f(g), f(g)\rangle d g<\infty .
$$


The right-regular representation on $L^{2}(\Gamma \backslash G, \chi)$ is easily seen to be unitary, and it is known that the right-regular representation decomposes discretely,

$$
R=\widehat{\bigoplus}_{\pi \in \overparen{G}} m(\pi) \pi,
$$

with finite multiplicities $m(\pi) \in \mathbb{N}$. This decomposition allows us to derive the Selberg trace formula: For $f \in C_{c}^{\infty}(G)$ we consider the operator $R(f)$ on $L^{2}(\Gamma \backslash G, \chi)$ given by

$$
R(f) \varphi(x)=\int_{G} f(g) \varphi(x g) d x,
$$

where $\varphi \in L^{2}(\Gamma \backslash G, \chi)$ and $d x$ is a $G$-invariant Radon measure on $\Gamma \backslash G$. The operator $R(f)$ is an integral operator, with integral kernel given by

$$
k_{f}(x, y)=\sum_{\gamma \in \Gamma} f\left(x^{-1} \gamma y\right) \chi(\gamma) .
$$

The operator $R(f)$ is of trace class and the trace can be computed as the integral

$$
\operatorname{tr} R(f)=\int_{\Gamma \backslash G} \sum_{\gamma \in \Gamma} f\left(x^{-1} \gamma x\right) \operatorname{tr} \chi(\gamma) d x .
$$

After some computation and reordering this sum with respect to the conjugacy classes $[\gamma]$ of $\Gamma$ one obtains

$$
\operatorname{tr} R(f)=\sum_{[\gamma]} \int \operatorname{vol}\left(\Gamma_{\gamma} \backslash G_{\gamma}\right) \mathcal{O}_{\gamma}(f) \operatorname{tr} \chi(\gamma) .
$$

In the above expression $\Gamma_{\gamma}, G_{\gamma}$ is the centralizer of $\gamma$ in $\Gamma$ and $G$ respectively. The orbital integral $\mathcal{O}_{\gamma}(f)$ is given by

$$
\mathcal{O}_{\gamma}(f)=\int_{G_{\gamma} \backslash G} f\left(x^{-1} \gamma x\right) d x .
$$

On the other hand we can compute the trace of $R(f)$ using the decomposition in (11) and obtain

$$
\operatorname{tr} R(f)=\sum_{\pi \in \widehat{G}} m(\pi) \operatorname{tr} \pi(f) .
$$


Thus, by equating (2) and (3) one obtains the Selberg trace formula

$$
\sum_{\pi \in \widehat{G}} m(\pi) \operatorname{tr} \pi(f)=\sum_{[\gamma]} \int \operatorname{vol}\left(\Gamma_{\gamma} \backslash G_{\gamma}\right) \mathcal{O}_{\gamma}(f) \operatorname{tr} \chi(\gamma)
$$

which connects spectral data (left-hand side) to geometric data (right-hand side) of $\Gamma \backslash G$.

In contrast to the above explanations, we will in this work allow $\chi: G \rightarrow$ $\mathrm{GL}(V)$ to be an arbitrary complex and finite dimensional representation, not necessarily unitary. If we follow the approach, which we have sketched for a unitary representation $\chi$, the first obstacle appearing, is the question how to define $L^{2}(\Gamma \backslash G, \chi)$. A satisfying answer will be given in Definition [2.2. But nevertheless, we will lose the unitarity of the right-regular representation $R$ and consequently, a decomposition (1) as in the unitary case will in general not hold. But under the conditions, that will be formulated in the subsequent section, it is shown in Proposition 3.15 that the right-regular representation on $L^{2}(\Gamma \backslash G, \chi)$ admits an increasing and exhausting filtration

$$
0=V_{0} \subset V_{1} \subset \cdots \subset \bigcup_{i=0}^{\infty} V_{i}=L^{2}(\Gamma \backslash G, \chi),
$$

such that the natural representation on $V_{i} / V_{i-1}$ is admissible and irreducible. The above filtration gives the means to derive a trace formula in Theorem 4.2 . The computation of the geometric side is completely analogous to the unitary case, whereas the spectral side can be computed using the filtration (4). We use the fact, that the trace of an operator on $L^{2}(\Gamma \backslash G, \chi)$, which is compatible with the filtration, can be computed by summing the individual traces of the induced operators on the graded parts $V_{i} / V_{i-1}$.

\section{Preliminaries}

In this work, let $G$ be a connected, semisimple Lie-group with finite center and $K$ a maximal compact subgroup of $G$. Let $\Gamma \subset G$ be a torsion free, uniform lattice. Fix a Haar measure $d g$, which is both a left- and right Haar measure, due to the existence of the uniform lattice $\Gamma$. We let $X:=\Gamma \backslash G$ be the 
compact quotient space with $G$-invariant measure $d x$, such that for $f \in C_{c}(G)$ the integral formula

$$
\int_{G} f(g) d g=\sum_{\gamma \in \Gamma} \int_{X} f(\gamma x) d x
$$

holds.

Definition 2.1. $\quad$ - For a complex vector space $V$ we let $\operatorname{GL}(V)$ be the group of all automorphisms of $V$. A representation $(\pi, V)$ of $G$ is a group homomorphism $\pi: G \rightarrow \mathrm{GL}(V)$.

- For a complex Hilbert space we let $\mathrm{GL}(H)$ be the group of all bijective and bounded endomorphisms of $H$. A (continuous) representation $(\pi, H)$ of $G$ is a group homomorphism $\pi: G \rightarrow \mathrm{GL}(H)$, such that the map

$$
\begin{aligned}
G \times H & \rightarrow H, \\
(g, v) & \mapsto \pi(g) v,
\end{aligned}
$$

is continuous. A continuous representation $\pi$ is said to be admissible if $\pi$ restricted to $K$ is unitary and each $\tau \in \widehat{K}$ occurs with only finite multiplicity. When the underlying representation space is a Hilbert space we will always mean a continuous representation without mentioning it anymore.

- A (Lie algebra) representation $(\pi, V)$ of the Lie algebra $\mathfrak{g}$ of $G$ is a complex vector space $V$ together with a Lie algebra homomorphism

$$
\begin{aligned}
\mathfrak{g} & \rightarrow \mathfrak{g l}(V), \\
X & \mapsto \pi(X) .
\end{aligned}
$$

- A $(\mathfrak{g}, K)$-module is a vector space $V$ which is both a Lie algebra representation of $\mathfrak{g}$ and a group representation of $K$ such that the representations are compatible in the following way: 
1. for any $v \in V, k \in K$ and $X \in \mathfrak{g}$

$$
k \cdot(X \cdot v)=(\operatorname{Ad}(k) X) \cdot(k \cdot v),
$$

2. for any $v \in V$ and $Y \in \mathfrak{k}$

$$
\left.\left(\frac{d}{d t} \exp (t Y) \cdot v\right)\right|_{t=0}=Y \cdot v
$$

The third condition ensures $K$-finiteness:

3. for any $v \in V$ the set $K v$ spans a finite-dimensional subspace of $V$.

Recall that the $K$-finite vectors of an admissible representation $(\pi, V)$ give rise to a $(\mathfrak{g}, K)$-module. Two admissible representations $\pi$ and $\eta$ are called equivalent, if the associated $(\mathfrak{g}, K)$-modules are isomorphic.

- The unitary dual $\widehat{G}$ of the group $G$ is the set of all irreducible unitary representations modulo unitary equivalence.

- The admissible dual $\widehat{G}_{\mathrm{adm}}$ of $G$ is the set of all irreducible admissible representations module admissible equivalence.

In the following $\chi$ will always be a finite dimensional complex representation of $\Gamma$, not necessarily unitary, with representation space $V=V_{\chi}$. Let $E=E_{\chi}$ be the associated vector bundle over $\Gamma \backslash G$. More precisely, we consider the bundle $\Gamma \backslash(G \times V)$, where $\Gamma$ acts on $G \times V$ via

$$
\gamma \cdot(g, v)=(\gamma g, \chi(\gamma) v)
$$

The image of $(g, v)$ under the canonical projection $G \times V \rightarrow E$ will be written as $\Gamma(g, v)$. Note, that $\Gamma(\gamma g, v)=\Gamma\left(g, \chi\left(\gamma^{-1}\right) v\right)$. Furthermore the group $G$ acts on $E$ via

$$
g \cdot \Gamma(h, v)=\Gamma\left(h g^{-1}, v\right) .
$$

There is a canonical identification of the smooth sections $\Gamma^{\infty}(X, E)$ of the bundle $E$ with the set of functions

$$
C^{\infty}(G, V)^{\Gamma}=\left\{f \in C^{\infty}(G, V): f(\gamma g)=\chi(\gamma) f(g) \text { for all } \gamma \in \Gamma, g \in G\right\}
$$


and we will freely switch between these two interpretations.

Definition 2.2. We choose any smooth hermitian metric $\langle\cdot, \cdot\rangle$ on $E$. If we choose a Haar-measure $d k$ on $K$ we can form the integral

$$
\int_{K}\langle\Gamma(g k, v), \Gamma(g k, w)\rangle_{\Gamma g k} d k
$$

This gives again a smooth hermitian fibre metric on $E$, which is $K$-equivariant and by replacing $\langle\cdot, \cdot\rangle$ with this one we can assume, that

$$
\langle\Gamma(g k, v), \Gamma(g k, w)\rangle_{\Gamma g k}=\langle\Gamma(g, v), \Gamma(g, w)\rangle_{\Gamma g}
$$

for arbitrary $g \in G, k \in K$ and $v, w \in V$.

Together with this smooth $K$-equivariant metric we obtain a pre-Hilbert space structure on the set of smooth sections $\Gamma^{\infty}(X, E)$ via

$$
(f, g):=\int_{\Gamma \backslash G}\langle f(x), g(x)\rangle_{x} d x
$$

We complete $\Gamma^{\infty}(X, E)$ with respect to the induced norm and obtain the Hilbert space of square integrable sections $L^{2}(X, E)$. The definition of $L^{2}(X, E)$ is independent of the chosen smooth fibre metric on $E$, since by compactness of $\Gamma \backslash G$ and the finite-dimensionality of the fibres, another smooth metric induces an equivalent norm on $\Gamma^{\infty}(X, E)$.

Definition 2.3. On $\Gamma^{\infty}(X, E)$ we define the right regular representation $R$ of $G$ as

$$
R(g) f(x):=g \cdot f(x g),
$$

where $f \in \Gamma^{\infty}(X, E), x \in X$ and $g \in G$.

If we use the identification of $\Gamma^{\infty}(X, E)$ with $C^{\infty}\left(G, V_{\chi}\right)^{\Gamma}$ the right regular representation for elements $f \in C^{\infty}\left(G, V_{\chi}\right)^{\Gamma}$ is just given by

$$
R(g) f(x)=f(x g)
$$

In the following paragraph we will show, that the right regular representation $R$ is continuous. We start out with the following proposition. 
Proposition 2.4. There exists a continuous function $\psi$ on $G$, such that

$$
\langle\Gamma(g h, v), \Gamma(g h, v)\rangle_{\Gamma g h} \leq \psi(h)\langle\Gamma(g, v), \Gamma(g, v)\rangle_{\Gamma g},
$$

for arbitrary $g, h \in G$ and $v \in V$. In particular, there exists for each compact set $C \subset G$ a constant $M$ depending only on $C$, such that $\langle\Gamma(g h, v), \Gamma(g h, v)\rangle_{\Gamma g h} \leq$ $M\langle\Gamma(g, v), \Gamma(g, v)\rangle_{\Gamma g}$, for all $h \in C$ and arbitrary $g \in G, v \in V$.

Proof. For every $h \in G$, there exists a contionuous section $A_{h} \in \Gamma(X, \operatorname{Hom}(E, E))$, such that for $v \in V$ we have

$$
\langle\Gamma(g h, v), \Gamma(g h, v)\rangle_{\Gamma g h}=\left\langle\Gamma\left(g, A_{h}(\Gamma g) v\right), \Gamma\left(g, A_{h}(\Gamma g) v\right)\right\rangle_{\Gamma g}
$$

As the metric is smooth, the dependence of $A_{h}$ on $h$ is smooth, in particular continuous. We let

$$
\left\|A_{h}(\Gamma g)\right\|_{\Gamma g}^{2}=\sup _{v \neq 0} \frac{\left\langle\Gamma\left(g, A_{h}(\Gamma g) v\right), \Gamma\left(g, A_{h}(\Gamma g) v\right)\right\rangle_{\Gamma g}}{\langle\Gamma(g, v), \Gamma(g, v)\rangle_{\Gamma g}} .
$$

Then we define $\psi(h)=\max _{\Gamma g \in \Gamma \backslash G}\left\|A_{h}(\Gamma g)\right\|_{\Gamma g}^{2}$ which satisfies the conditions of the proposition.

We choose once and for all a representative $\left(\tau, V_{\tau}\right)$ for each class in $\widehat{K}$, the unitary dual of $K$. For a unitary representation $(\pi, V)$ of $K$ we let $V(\tau)$ be the $\tau$-isotype. Recall the following theorem:

Theorem 2.5. [1, Theorem 7.3.2.] For $(\pi, V)$ a unitary representation of $K$ the representation space is a direct Hilbert space sum of all the $K$-isotypes:

$$
V=\widehat{\bigoplus}_{\tau \in \widehat{K}} V(\tau)
$$

Proposition 2.6. The right regular representation $R$ on $\Gamma^{\infty}(X, E)$ is continuous with respect to the $L^{2}$-topology. In particular it extends to a continuous representation of $L^{2}(X, E)$. The restriction of $R$ to the maximal compact subgroup $K$ is unitary and hence we get a $K$-isotypical decomposition

$$
L^{2}(X, E)=\widehat{\bigoplus}_{\tau \in \widehat{K}} L^{2}(X, E)(\tau)
$$


Proof. Let $C \subset G$ be a compact subset and $h \in C$. Let $M$ be as in Proposition 2.4. Then for $f \in \Gamma^{\infty}(X, E)$ we estimate

$$
\begin{aligned}
(R(h) f, R(h) f) & =\int_{X}\langle h \cdot f(x h), h \cdot f(x h)\rangle_{x} d x \\
& \leq \int_{X} M\langle f(x), f(x)\rangle_{x} d x \\
& =M(f, f),
\end{aligned}
$$

independent of $h \in C$. Hence the operator norm of $R(h)$ is uniformally bounded on each compact subset $C \subset G$. Since for fixed $f \in \Gamma^{\infty}(X, E)$ the map

$$
\begin{aligned}
G & \rightarrow L^{2}(X, E) \\
g & \mapsto R(g) f
\end{aligned}
$$

is continous, the continuity of the right-regular representation $R$ follows. The unitarity as a representation of $K$ is clear from the $K$-equivariance of the fibre metric: If we let $k \in K$ then we get

$$
\begin{aligned}
(R(k) f, R(k) f) & =\int_{X}\langle k \cdot f(x k), k \cdot f(x k)\rangle_{x} d x \\
& =\int_{X}\langle f(x k), f(x k)\rangle_{x k} d x \\
& =\int_{X}\langle f(x), f(x)\rangle_{x} d x \\
& =(f, f) .
\end{aligned}
$$

\section{The spectral decomposition of the Casimir operator}

Definition 3.1. We let $\mathfrak{g}, \mathfrak{g}_{\mathbb{C}}, U\left(\mathfrak{g}_{\mathbb{C}}\right), Z\left(\mathfrak{g}_{\mathbb{C}}\right)$ be the Lie-algebra of $G$, its complexification, the universal envelopping algebra, as well as its center, respectively. We endow $G / K$ with the $G$-invariant metric induced by the Killing form $\langle\cdot, \cdot\rangle$. The Killing form is non-degenerate, and hence it gives an identification of $\mathfrak{g}$ and its dual space $\mathfrak{g}^{*}$. If $X_{1}, \ldots, X_{n}$ is a basis of $\mathfrak{g}$, then we define the 
Casimir element as

$$
\Omega=Y_{1} X_{1}+\ldots Y_{n} X_{n} \in U\left(\mathfrak{g}_{\mathbb{C}}\right)
$$

where $Y_{1}, \ldots, Y_{n}$ is a dual basis of $X_{1}, \ldots, X_{n}$ with respect to the Killing form. The definition of $\Omega$ is independent of the chosen orthonormal basis and $\Omega \epsilon$ $Z\left(\mathfrak{g}_{\mathbb{C}}\right)$ [2, Proposition 8.6.].

If $\mathfrak{g}=\mathfrak{k} \oplus \mathfrak{p}$ is the Cartan decomposition of $\mathfrak{g}$ and $\theta$ is the Cartan involution on $\mathfrak{g}$ the map

$$
(X, Y) \mapsto-\langle X, \theta(Y)\rangle
$$

is a positive definite bilinear form. If $X_{1}, \ldots, X_{l}$ is an orthonormal basis of $\mathfrak{k}$ and $Y_{1}, \ldots, Y_{k}$ an orthonormal basis of $\mathfrak{p}$ with respect to the above bilinear form, we find

$$
\begin{aligned}
\Omega & =-X_{1}^{2}-\cdots-X_{l}^{2}+Y_{1}^{2}+\cdots+Y_{k}^{2} \\
& =\Omega_{K}+Y_{1}^{2}+\cdots+Y_{k}^{2},
\end{aligned}
$$

where $\Omega_{K}$ is the Casimir element of $U\left(\mathfrak{k}_{\mathbb{C}}\right)$.

We will need the following results, to deduce a nice spectral decomposition of the Casimir operator on $L^{2}(X, E)$.

Theorem 3.2. [4, Theorem 8.4.] Let $M$ be a closed manifold and $D$ an elliptic differential operator on a metric bundle $\mathcal{E}$. If the resolvent set $\rho(D) \neq \varnothing$ is not empty, the spectrum $\sigma(D)$ is discrete and for each $\lambda \in \sigma(D)$ there exists a decomposition $L^{2}(M, \mathcal{E})=E_{\lambda} \oplus E_{\lambda}^{\prime}$, such that

1. $E_{\lambda} \subset \Gamma^{\infty}(M, \mathcal{E}), \operatorname{dim} E_{\lambda}<\infty$, and $E_{\lambda}$ is invariant under $D$ and there exists some $n>0$, such that $(D-\lambda)^{n} E_{\lambda}=0$,

2. $E_{\lambda}^{\prime}$ is a closed subspace of $L^{2}(M, \mathcal{E})$ invariant under $D$. If we denote by $D_{\lambda}$ the restriction of $D$ to $E_{\lambda}^{\prime}$, then $\lambda \notin \sigma\left(A_{\lambda}\right)$.

Theorem 3.3. [4, Theorem 8.4.] Let $M$ be a closed manifold and $D$ an elliptic differential operator on a metric bundle $\mathcal{E}$. For an interval I we define the cone

$$
\Lambda_{I}=\left\{r e^{i \theta}: 0 \leq r<\infty, \theta \in I\right\} .
$$


For $\varepsilon>0$ there exists an $R>0$ such that the spectrum $\sigma(D)$ is contained in the set $B_{R}(0) \cup \Lambda_{[-\varepsilon, \varepsilon]}$.

Definition 3.4. Let $\mathcal{E}$ be a vector bundle over a Riemannian manifold $M$. A second order differential operator $D$ is a Laplace type operator if for the principal symbol

$$
\sigma_{2}(D)(x, \xi)=\|\xi\|^{2}
$$

for arbitrary $x \in M$ and $\xi \in T^{*} M$. In particular each Laplace type operator is elliptic.

The reason why we introduce the notion of Laplace type operators, is that a reasonable spectral theory can be developed for those.

Proposition 3.5. The Casimir element $\Omega$ induces on each $K$-isotype $L^{2}(X, E)(\tau)$ a Laplace type operator, having discrete spectrum. We will denote the induced operator by $\Omega_{\tau}$. Let

$$
V_{\tau, \lambda}:=\left\{f \in L^{2}(X, E)(\tau):\left(\Omega_{\tau}-\lambda\right)^{n} f=0 \text { for some } n \in \mathbb{N}\right\}
$$

the generalized eigenspace belonging to $\lambda \in \operatorname{spec}\left(\Omega_{\tau}\right)$. Then $V_{\tau, \lambda} \subset \Gamma^{\infty}(X, E)(\tau)$, $\operatorname{dim} V_{\tau, \lambda}<\infty$ and $V_{\tau, \infty}$ is stable under $K$ as well as $Z\left(\mathfrak{g}_{\mathbb{C}}\right)$.

Proof. We let $E_{\chi, \tau}$ be the vector bundle $G \times_{\Gamma \times K} V_{\chi} \otimes V_{\tau}$ with base space $\Gamma \backslash G / K$ where $\Gamma \times K$ acts on $G \times V_{\chi} \otimes V_{\tau}$ via

$$
(\gamma, k) \cdot(g, v \times w)=\left(\gamma g k^{-1}, \chi(\gamma) v \times \tau(k) w\right)
$$

Let $X \in \mathfrak{g}$ and $f \in C^{\infty}\left(G, V_{\chi} \times V_{\tau}\right)$. X induces a differential operator via

$$
X f(g)=\left.\frac{d}{d t} f(g \exp (t X))\right|_{t=0}
$$

and this map from the Lie algebra to the algebra of differential operators extends to the universal envelopping algebra $U\left(\mathfrak{g}_{\mathbb{C}}\right)$. From the definition it is easily seen, that the induced differential operator is left invariant, meaning that

$$
X\left(L_{h}(f)\right)(g)=(X f)(h g),
$$


where $L_{h}$ is the left translation by the element $h$. On the other hand, we find for $X \in \mathfrak{g}$ and the right translation $R_{h}$ and any element $h \in G$, that

$$
\begin{aligned}
X\left(R_{h}(f)\right)(g) & =\left.\frac{d}{d t} f(g \exp (t X) h)\right|_{t=0} \\
& =\left.\frac{d}{d t} f(g h \exp (\operatorname{Ad}(h) t X))\right|_{t=0} \\
& =(\operatorname{Ad}(h) X)(f)(g h) .
\end{aligned}
$$

Thus we get $X\left(R_{h}(f)\right)(g)=(\operatorname{Ad}(h) X)(f)(g h)$ for all $X \in U\left(\mathfrak{g}_{\mathbb{C}}\right)$. Since $\Omega \in Z\left(\mathfrak{g}_{\mathbb{C}}\right)$ this yields

$$
\Omega\left(R_{h}(f)\right)(g)=(\Omega f)(g h),
$$

for arbitrary $g, h \in G$ and consequently we have for $f \in C^{\infty}\left(G, V_{\chi} \otimes V_{\tau}\right)^{\Gamma \times K}$ that

$$
(\Omega f)\left(\gamma x k^{-1}\right)=\chi(\gamma) \otimes \tau(k) \Omega f(x)
$$

and thus $C^{\infty}\left(G, V_{\chi} \otimes V_{\tau}\right)^{\Gamma \times K}$ is stable under $\Omega$.

Furthermore

$$
\Gamma^{\infty}(X, E)(\tau) \cong V_{\tau} \otimes \operatorname{Hom}_{K}\left(V_{\tau}, \Gamma^{\infty}(X, E)\right)
$$

and

$$
\begin{aligned}
\operatorname{Hom}_{K}\left(V_{\tau}, \Gamma^{\infty}(X, E)\right) & \cong\left(\Gamma^{\infty}(X, E) \otimes V_{\tau}\right)^{K} \\
& \cong\left(C^{\infty}(G) \otimes V_{\chi} \otimes V_{\tau}\right)^{\Gamma \times K} \\
& \cong \Gamma^{\infty}\left(\Gamma \backslash G / K, E_{\chi, \tau}\right) .
\end{aligned}
$$

Hence Id $\otimes \Omega$ induces an operator $\Omega_{\tau}$ on $L^{2}(E)(\tau)$.

Next we will show, that it is a Laplace type operator.

Consider the Cartan decomposition $\mathfrak{g}=\mathfrak{k} \oplus \mathfrak{p}$ and basises $X_{1}, \ldots X_{l}$ and $Y_{1}, \ldots Y_{k}$ as before, such that

$$
\Omega=-X_{1}^{2}-\cdots-X_{l}^{2}+Y_{1}^{2}+\cdots+Y_{k}^{2}=\Omega_{K}+Y_{1}^{2}+\cdots+Y_{k}^{2} .
$$

For $\Gamma g K \in \Gamma \backslash G / K$ there exists a neighbourhood $U$, such that we can choose the map

$$
g \exp \left(y_{1} Y_{1}+\cdots+y_{k} Y_{n}\right) \mapsto\left(y_{1}, \ldots, y_{k}\right)
$$


as a local coordinate map on $U$. Inside $U$ and with these coordinates we find

$$
(\Omega f)(g)=\sum \frac{\partial^{2}}{\partial y_{i}^{2}} f(g)+\operatorname{Id} \otimes \sum(d \tau)\left(\Omega_{K}\right) f(g) .
$$

But since the representation $V_{\tau}$ is irreducible and $\Omega_{K} \in Z\left(\mathfrak{k}_{\mathbb{C}}\right)$ the operator $(d \tau)\left(\Omega_{K}\right)$ acts as a scalar, according to the Lemma of Schur. Consequently $\Omega$ induces a second order differential operator with principal symbol $\left(\Omega_{\tau}\right)(x, \xi)=$ $\|\xi\|^{2}$.

From Theorem 3.3 and 3.2 it is now clear, that the spectrum is discrete. By Theorem 3.2 it follows also, that the generalized eigenspace $V_{\tau, \lambda}$ is finite dimensional and $V_{\tau, \lambda} \subset \Gamma^{\infty}(X, E)(\tau)$.

$V_{\tau, \lambda}$ is stable under $K$, since $K \cdot L^{2}(X, E)(\tau) \subset L^{2}(X, E)(\tau)$ and $\operatorname{Ad}(k) \Omega=\Omega$. Since $Z\left(\mathfrak{g}_{\mathbb{C}}\right) \cdot \Gamma^{\infty}(X, E)(\tau) \subset \Gamma^{\infty}(X, E)(\tau)$ it is also clear, that $Z\left(\mathfrak{g}_{\mathbb{C}}\right) \cdot V_{\tau, \lambda} \subset$ $V_{\tau, \lambda}$

Proposition 3.6. The space $L^{2}(X, E)(\tau)$ is the closure of the algebraic direct sum of all generalized eigenspaces:

$$
L^{2}(X, E)(\tau)=\bigoplus_{\lambda \in \sigma\left(\Omega_{\tau}\right)} V_{\tau, \lambda} .
$$

To prepare the proof of Proposition 3.6 we need:

Definition 3.7. Let $H$ be a Hilbert space and $G$ a linear operator with nonempty resolvent set $\rho(G) \neq \varnothing$. An operator $B$ is said to be compact relative to $G$ if $D(G) \subset D(B)$ and the operator $B R_{\lambda}(G)$ is compact, where $R_{\lambda}(G)=$ $(G-\lambda)^{-1}$ is the resolvent of $G$.

Theorem 3.8. [3, Theorem 4.3.] Let $H$ be a Hilbert space and $G$ a self-adjoint operator. The resolvent $R_{\lambda}(G)$ is assumed to be a Schatten class operator and $B$ an operator relatively compact to $G$. Then the operator $C=G+B$ has a compact resolvent and $H$ is the closure of the generalized eigenspaces of $C$ :

$$
H=\bigoplus_{\lambda \in \sigma(C)} V_{\lambda}
$$

Proof of Proposition 3.6. According to Proposition 3.5 we have $\sigma\left(\Omega_{\tau}\right)(x, \xi)=$ $\|\xi\|^{2}$. Hence we get $\Omega_{\tau}=\Delta+B$, where $\Delta$ is the Bochner-Laplace operator, 
which is self-adjoint, and $B$ is a first order differential operator. The resolvent $R_{\lambda}(\Delta)$ is of order -2 , hence compact and a Schatten class operator. Similarly $B R_{\lambda}(\Delta)$ is of order -1 and also compact. The statement now follows by applying Theorem 3.8 .

Definition 3.9. Let $V_{\text {fin }} \subset \Gamma^{\infty}(X, E)$ be the set of all smooth sections which are $K$ - as well as $Z\left(\mathfrak{g}_{\mathbb{C}}\right)$-finite.

Proposition 3.10. $V_{\text {fin }}$ is the algebraic direct sum of all generalized eigenspaces for the operators $\Omega_{\tau}$ :

$$
V_{\text {fin }}=\bigoplus_{\tau \in \widehat{K}} \bigoplus_{\lambda \in \sigma\left(\Omega_{\tau}\right)} V_{\tau, \lambda} .
$$

In particular, $V_{\text {fin }}$ is dense in $L^{2}(X, E)$ and consequently, because $V_{\text {fin }} \subset \Gamma^{\infty}(X, E)$, it is dense in $\Gamma^{\infty}(X, E)$.

Proof. If $f$ is an element in the above direct sum, it is clear that $f \in V_{\text {fin }}$, since each generalized eigenspace $V_{\tau, \lambda}$ is finite dimensional and $K$ - and $Z\left(\mathfrak{g}_{\mathbb{C}}\right)$ invariant.

If we now take $f \in V_{\text {fin }}$ we obtain

$$
f \in \bigoplus_{\tau \in \widehat{K}} L^{2}(X, E)(\tau)
$$

because of the $K$-finiteness of $f$. Hence we can assume, that $f \in L^{2}(X, E)(\tau)$ for some $\tau \in \widehat{K}$. Let $W \subset L^{2}(X, E)(\tau)$ be the finite-dimensional $Z\left(\mathfrak{g}_{\mathbb{C}}\right)$ - and $K$ invariant vectorspace, generated by $f$. $W$ is stable under $\Omega_{\tau}$, since this operator is induced by $\Omega \in Z\left(\mathfrak{g}_{\mathbb{C}}\right)$. Consider the operator $\left.\Omega_{\tau}\right|_{W}$. By the theorem about the Jordan normal form we have a direct sum decomposition

$$
W=\bigoplus_{\lambda \in \sigma\left(\Omega_{\tau}\right)} V_{\tau, \lambda} \cap W
$$

This proves, that $f$ lies in the above direct sum.

We will now cite two propositions, which we will need to infer a filtration of the $(\mathfrak{g}, K)$-module $V_{\text {fin }}$.

Proposition 3.11. [5, Corollary 3.4.7.] Let $V$ a finitely generated $(\mathfrak{g}, K)$ module, such that $\operatorname{dim} Z\left(\mathfrak{g}_{\mathbb{C}}\right) v<\infty$ for all $v \in V$. Then $V$ is admissible. 
Proposition 3.12. [2, Corollary 10.42.] Each Harish-Chandra-module V (in other words: a finitely generated, admissible $(\mathfrak{g}, K)$-module) has a finite composition series

$$
V=W_{k} \supset W_{k-1} \supset \cdots \supset W_{0}=0
$$

with irreducible quotients $W_{j} / W_{j-1}$. The multiplicities of the irreducible subquotients are independent of the chosen composition series.

Proposition 3.13. The exists a seperated, exhaustive and increasing filtration Fil $i$, where $i$ ranges over all nonnegative integers, of $V_{\text {fin }}$ as a $(\mathfrak{g}, K)$-module, such that each quotient Fil $V / F i l_{i-1} V$ is admissible and irreducible. The graduated module

$$
\operatorname{Gr} V_{\text {fin }}=\bigoplus_{i=0}^{\infty} F i l_{i+1} V / F i l_{i} V
$$

is independent of the chosen filtration.

Proof. We choose a generalized eigenspace $V_{\tau, \lambda}$ from the direct sum in (5). Since it is $K$ - and $Z\left(\mathfrak{g}_{\mathbb{C}}\right)$-stable, we find $U\left(\mathfrak{g}_{\mathbb{C}}\right) V_{\tau, \lambda} \subset V_{\text {fin }}$. According to Proposition 3.11 the $(\mathfrak{g}, K)$-module $U\left(\mathfrak{g}_{\mathbb{C}}\right) V_{\tau, \lambda}$ is admissible and according to Proposition 3.12 there exists a finite composition series

$$
U\left(\mathfrak{g}_{\mathbb{C}}\right) V_{\tau, \lambda}=\mathrm{Fil}_{k} V \supset \mathrm{Fil}_{k-1} V \supset \cdots \supset \mathrm{Fil}_{0} V=0
$$

such that $\bigoplus_{i=1}^{k} \mathrm{Fil}_{i} V / \mathrm{Fil}_{i-1} V$ is independent of the chosen composition series. Now proceed in the same manner with $V_{\text {fin }} / U\left(\mathfrak{g}_{\mathbb{C}}\right) V_{\tau, \lambda}$ to obtain the filtration.

Proposition 3.14. Each element $f \in V_{\tau, \lambda}$ is real-analytic.

Proof. If $f \in V_{\tau, \lambda}$ there exists some $N \in \mathbb{N}$, such that $\left(\Omega_{\tau}-\lambda\right)^{N} f=0$. Hence $f$ is annihilated by the elliptic differential operator $\left(\Omega_{\tau}-\lambda\right)^{N}$, whence it is real-analytic.

The filtration of $V_{\text {fin }}$ furnishes a filtration of the right regular representation, as we will show now.

Proposition 3.15. The representation $\left(R, L^{2}(X, E)\right)$ admits a seperated, exhaustive and increasing filtration of subrepresentations

$$
0=V_{0} \subset V_{1} \subset \cdots \subset \bigcup_{i=0}^{\infty} V_{i}=L^{2}(X, E),
$$


induced by the filtration of the $(\mathfrak{g}, K)$-module $V_{\text {fin }}$ from Proposition 3.13.

Proof. We let $V_{i}=\overline{\mathrm{Fil}_{i} V}$ be the closure of $\operatorname{Fil}_{i} V$ in $L^{2}(X, E)$. It is easy to see, that $V_{i}$ is stable under $G$. To show this, it is enough to prove $G \cdot \operatorname{Fil}_{i} V \subset V_{i}$, since the representation is continuous. Let $h \in V_{i}^{\perp}$. For $f \in \operatorname{Fil}_{i} V \subset V_{\text {fin }}$ the function

$$
g \mapsto(R(g) h, f)
$$

is real-analytic according to Proposition 3 , For $X \in \mathfrak{g}$ on a sufficient small neighbourhood of 0 , we have a Taylor expansion:

$$
\begin{aligned}
\langle R(\exp X) h, f\rangle & =\left.\sum_{n=0}^{\infty} \frac{1}{n !} X^{n}\langle R(g) h, f\rangle\right|_{g=1} \\
& =\sum_{n=0}^{\infty} \frac{1}{n !}\left\langle X^{n} h, f\right\rangle .
\end{aligned}
$$

Since $h \in \operatorname{Fil}_{i} V$, hence also $X^{n} h \in \operatorname{Fil}_{i} V$ we get $\langle R(\exp X) h, f\rangle=0$. But then $\langle R(g) h, f\rangle=0$ in a neighbourhood of 1 , and because of analycity, on the whole of $G$. Since $h \in \operatorname{Fil}_{i} V$ and $f \in V_{i}^{\perp}$ were arbitrary we get $G \cdot \operatorname{Fil}_{i} V \subset V_{i}$.

For each $i \in \mathbb{N}$ we obtain the quotient representation on $V_{i} / V_{i-1}$, which we will denote by $R_{i}$.

Proposition 3.16. For the $K$-finite vectors of $R_{i}$ we obtain

$$
\left(V_{i} / V_{i-1}\right)_{R_{i}, K} \cong F i l_{i} V / F i l_{i-1} V .
$$

Proof. This is is clear, because $R_{i}$ is admissible, and so $\left(V_{i} / V_{i-1}\right)(\tau)$ is finite dimensional, but

$$
\left(\mathrm{Fil}_{i} V / \mathrm{Fil}_{i-1} V\right)(\tau) \subset\left(V_{i} / V_{i-1}\right)(\tau)
$$

is dense, and thus the both must be equal.

Together with the following theorem, it follows that the representations $R_{i}$ are irreducible:

Theorem 3.17. [5, Theorem 3.4.12.] Let $(\pi, H)$ be an admissible Hilbert-space representation of $G$. Then $(\pi, H)$ is irreducible, iff the associated $(\mathfrak{g}, K)$-module $H_{\pi, K}$ is irreducible. 


\section{The trace formula}

Definition 4.1. Let $(H,\langle\cdot, \cdot\rangle)$ be a Hilbert-space. A compact operator $T$ is of trace class, if $\sum_{i} s_{i}(T)<\infty$, where $s_{i}(T)$ denote the singular values of the operator $T$. Let $\left(e_{i}\right)_{i \in I}$ be an orthonormal basis of $H$. The trace of a trace class operator $T$ is defined as

$$
\operatorname{tr}(T)=\sum_{i \in I}\left\langle T e_{i}, e_{i}\right\rangle
$$

One can show that this sum converges absolutely and is independent of the chosen orthonormal basis $\left(e_{i}\right)_{i \in I}([\underline{1}$, Theorem 5.3.5]).

Now let $(\pi, H)$ be an admissible representation of $G$. The representation is said to be of trace class if for each $f \in C_{c}^{\infty}(G)$ the operator

$$
\pi(f)=\int_{G} f(g) \pi(g) d g
$$

is of trace class.

Theorem 4.2. Let $f \in C_{c}^{\infty}(G)$. The operator $R(f)$ on $L^{2}(X, E)$ is an integral operator with integral kernel

$$
k_{f}(x, y)=\sum_{\gamma \in \Gamma} f\left(x^{-1} \gamma y\right) \chi(\gamma)
$$

Thus $R(f)$ is of trace class and the following trace formula holds

$$
\sum_{[\gamma]} \operatorname{vol}\left(\Gamma_{\gamma} \backslash G_{\gamma}\right) \mathcal{O}_{\gamma}(f) \operatorname{tr} \chi(\gamma)=\sum_{\pi \in \widehat{G}_{a d m}} N_{\Gamma, \chi}(\pi) \operatorname{tr} \pi(f)
$$

where on the left hand side we sum over all conjugacy classes $[\gamma]$ of $\Gamma$ and $\mathcal{O}_{\gamma}(f)$ is the orbital integral

$$
\mathcal{O}_{\gamma}(f)=\int_{G_{\gamma} \backslash G} f\left(x^{-1} \gamma x\right) d x
$$

The natural number $N_{\Gamma, \chi}(\pi)$ denotes the multiplicity of the admissible and irreducible representation $\pi$ in $\widehat{\oplus}_{i=0}^{\infty} V_{i} / V_{i-1}$. 
Proof. Let $h \in C_{c}(G)$, such that $\sum_{\gamma \in \Gamma} h(\gamma x)=1$. For $\varphi \in L^{2}(X, E)$ we compute

$$
\begin{aligned}
R(f) \varphi(x) & =\int_{G} f(y) \varphi(x y) d y \\
& =\int_{G} f\left(x^{-1} y\right) \varphi(y) d y \\
& =\int_{G} \sum_{\gamma \in \Gamma} h\left(\gamma^{-1} y\right) f\left(x^{-1} y\right) \varphi(y) d y \\
& =\sum_{\gamma \in \Gamma} \int h(y) f\left(x^{-1} \gamma y\right) \varphi(\gamma y) d y \\
& =\int_{G} h(y) \sum_{\gamma \in \Gamma} f\left(x^{-1} \gamma y\right) \chi(\gamma) \varphi(y) d y \\
& =\int_{\Gamma \backslash G} \sum_{\gamma^{\prime} \in \Gamma} h\left(\gamma^{\prime} y\right) \sum_{\gamma \in \Gamma} f\left(x^{-1} \gamma \gamma^{\prime} y\right) \chi(\gamma) \varphi\left(\gamma^{\prime} y\right) d y \\
& =\int_{\Gamma \backslash G} \sum_{\gamma \in \Gamma} f\left(x^{-1} \gamma y\right) \chi(\gamma) \varphi(y) d y .
\end{aligned}
$$

This computation shows that $R(f)$ is an integral operator with integral kernel

$$
k_{f}(x, y)=\sum_{\gamma \in \Gamma} f\left(x^{-1} \gamma y\right) \chi(\gamma)
$$

Then we can compute the trace of $R(f)$ by integrating the kernel along the diagonal:

$$
\operatorname{tr} R(f)=\int_{X} \sum_{\gamma \in \Gamma} f\left(x^{-1} \gamma x\right) \operatorname{tr} \chi(\gamma) d x
$$


Breaking the integration up into the different conjugacy classes of $G$ we get

$$
\begin{aligned}
\operatorname{tr} R(f) & =\int_{\Gamma \backslash G} \sum_{\gamma^{\prime} \in \Gamma} h\left(\gamma^{\prime} x\right) \sum_{\gamma \in \Gamma} f\left(x^{-1} \gamma x\right) \operatorname{tr} \chi(\gamma) d x \\
& =h(x) \int_{G} \sum_{\gamma \in \Gamma} f\left(x^{-1} \gamma x\right) \operatorname{tr} \chi(\gamma) d x \\
& =\sum_{\gamma \in \Gamma} \int_{G} h(x) f\left(x^{-1} \gamma x\right) \operatorname{tr} \chi(\gamma) d x \\
& =\sum_{[\gamma]} \sum_{\sigma \in \Gamma_{\gamma} \backslash \Gamma} \int_{G} h\left(\sigma^{-1} x\right) f\left(x^{-1} \gamma x\right) \operatorname{tr} \chi(\gamma) d x \\
& =\sum_{[\gamma]} \int_{\Gamma_{\gamma} \backslash G} \sum_{\sigma \in \Gamma_{\gamma} \backslash \Gamma} \sum_{\eta \in \Gamma_{\gamma}} h\left(\sigma^{-1} \eta x\right) f\left(x^{-1} \gamma x\right) \operatorname{tr} \chi(\gamma) d x \\
& =\sum_{[\gamma]} \int_{\Gamma_{\gamma} \backslash G} f\left(x^{-1} \gamma x\right) \operatorname{tr} \chi(\gamma) d x \\
& =\sum_{[\gamma]} \int_{G_{\gamma} \backslash G} \int_{\Gamma_{\gamma} \backslash G_{\gamma}} f\left((\sigma x)^{-1} \gamma \sigma x\right) \operatorname{tr} \chi(\gamma) d \sigma d x \\
& =\operatorname{dim}\left(V_{\chi}\right) \operatorname{vol}(\Gamma \backslash G)+\sum_{[\gamma] \neq[1]} \operatorname{vol}\left(\Gamma_{\gamma} \backslash G_{\gamma}\right) \mathcal{O}_{\gamma}(f) \operatorname{tr} \chi(\gamma)
\end{aligned}
$$

On the other hand, according to Proposition 3.15 we have a filtration of the representation space $L^{2}(X, E)$

$$
0=V_{0} \subset V_{1} \subset \cdots \subset \bigcup_{i=0}^{\infty} V_{i}=L^{2}(X, E) .
$$

Thus, when $R_{i}$ is the by $R$ induced representation on $V_{i} / V_{i-1}$ we get

$$
\operatorname{tr} R(f)=\sum_{i=0}^{\infty} \operatorname{tr} R_{i}(f)
$$

but the right-hand term is obviously equal to

$$
\sum_{\pi \in \widehat{G}_{\mathrm{adm}}} N_{\Gamma, \chi}(\pi) \operatorname{tr} \pi
$$




\section{References}

[1] Anton Deitmar and Siegfried Echterhoff. Principles of harmonic analysis. Universitext. Springer, New York, 2009.

[2] Anthony W. Knapp. Representation theory of semisimple groups. Princeton Landmarks in Mathematics. Princeton University Press, Princeton, NJ, 2001. An overview based on examples, Reprint of the 1986 original.

[3] A. S. Markus. Introduction to the spectral theory of polynomial operator pencils, volume 71 of Translations of Mathematical Monographs. American Mathematical Society, Providence, RI, 1988. Translated from the Russian by H. H. McFaden, Translation edited by Ben Silver, With an appendix by M. V. Keldysh.

[4] M. A. Shubin. Pseudodifferential operators and spectral theory. SpringerVerlag, Berlin, second edition, 2001. Translated from the 1978 Russian original by Stig I. Andersson.

[5] Nolan R. Wallach. Real reductive groups. I, volume 132 of Pure and Applied Mathematics. Academic Press Inc., Boston, MA, 1988.

Anton Deitmar

Mathematisches Institut

Auf Der Morgenstelle 10

72076 TÜBINGEN

GERMANy

deitmar@uni-tuebingen.de

Frank Monheim

Mathematisches Institut

Auf Der Morgenstelle 10

72076 TÜBINGEN

Germany

frank.monheim@uni-tuebingen.de 